\title{
Biotemplating of a Highly Porous Cellulose-Silica Composite from Apium graveolens by a Low-Toxicity Sol-Gel Technique
}

\author{
MAX MROZ, ${ }^{1}$ MUHAMMED ALI, ${ }^{2}$ JERRY HOWARD ${ }^{2}$ \\ KRISTA CARLSON ${ }^{2}$ and STEVEN E. NALEWAY (i) ${ }^{1,3}$ \\ 1.-Department of Mechanical Engineering, University of Utah, Salt Lake City, UT, USA. \\ 2.-Department of Materials Science and Engineering, University of \\ Utah, Salt Lake City, UT, USA. 3.—e-mail: steven.naleway@mech.utah.edu
}

A sol-gel biotemplating technique for the creation of a cellulose-silica composite from Apium graveolens (Pascal celery) has been investigated. The solgel biotemplating technique was inspired by $\mathrm{pH}$ catalyzed methods used for the creation of hydrogels. This technique did not require the use of highly toxic chemicals or an elevated temperature, and therefore is more environmentally friendly than existing biotemplating techniques. The resulting cellulose-silica composites were geometrically similar to the templated live celery, demonstrating permeability under gas flow at elevated pressure, and the elastic modulus and ultimate compressive strength (UCS) increased by $15.05 \times 10^{4} \%$ and $3880 \%$, respectively, when compared to live celery. The lack of toxic chemicals or elevated temperature, and the dramatic increase in modulus and UCS provide a low-toxicity alternative to harness the complex and multiscale structure and porosity of organic tissues in bioinspired materials.

\section{INTRODUCTION}

Porous silica materials possess properties useful for a range of applications, including drug delivery, ${ }^{1}$ carbon capture, ${ }^{2}$ and catalysis. ${ }^{3}$ The composition and structure of these materials is responsible for their many applications due to their high surface area and framework-like structural composition. ${ }^{4}$ Manufacturing methods for fabricating porous silica materials can generally be categorized as either bottom-up self-assembly ${ }^{2,3,5}$ or templating. ${ }^{6}$ Bottom-up self-assembly techniques are typically used to create mesoporous silica materials, or materials characterized by pore sizes confined to the nanoscale. $^{5}$ This differs from templating techniques, which are used to create macroporous silica materials, or materials characterized by pore sizes on the macro- and microscale. ${ }^{7,8}$ The functionality of

(Received October 26, 2020; accepted March 25, 2021;

published online May 6, 2021) porous silica materials can be improved through the creation of an ordered porous structure containing pores at multiple length scales. ${ }^{3,5,7,8}$

The importance of multiscale porosity and morphology to functionality is illustrated by biological materials, which are composed of weak constituents that outperform unstructured synthetic materials of the same constituent composition in both mechanical and functional properties. ${ }^{6,9,10}$ For example, a gradient in porosity along the cross-sections of vascular plants provides strength while minimizing material, ${ }^{11,12}$ and a gradient in vessel diameter along the plant's height minimizes hydraulic resistance. ${ }^{13}$ To capture the properties of biological materials by duplicating their structure in an inorganic material, a subset of templating techniques, known as biotemplating, has emerged. ${ }^{6,14,15}$ Similar techniques have been described using various terminology, including biomorphous materials and biomorphic mineralization. ${ }^{14,15}$ Therefore, for the purposes of this article, the working definition of biotemplating is: the direct transformation of a biological material into a positive replica of the original structure in a new material, as defined in Refs. 6 and 14. 
Biotemplating is a three-step process ${ }^{6,13}$ in which (1) the organic template is prepared (e.g., chemically removing constituents of the biological structure $\left.{ }^{16,17}\right)$, (2) the inorganic material is deposited into or onto the organic template, and (3) the organic template is removed (e.g., thermally ${ }^{17}$ or chemically ${ }^{15}$ ). Biotemplating for the creation of highly ordered porous silica materials has relied primarily on plants as templates, due to their cellular structure, ability to withstand the templating process, and the wide range of functional properties found in plants. ${ }^{6,17,18}$ These properties range from fluid transport ${ }^{18}$ to actuation, ${ }^{19}$ and are typically the result of the material's structuring ${ }^{17-19}$ (i.e., the result of passive systems, not controlled by living cells).

Previous macroscale biotemplating work for silica materials has focused on templating functional structures found in woody plants high in lignin. ${ }^{17}$ These plants are used because of the toughness of the lignin, which can withstand template preparation without the erosion of their microstructure. ${ }^{6,20}$ These techniques have utilized a sol-gel process with an alkoxide precursor such as tetramethyl orthosilicate in which a plant structure was partially eroded in the preparation phase (e.g., using cyclohexane in a Soxhlet evaporator). The material was subsequently infiltrated with a liquid precursor solution, and the solution gelled when reacted with a catalyst. These steps were followed by the removal of the remaining biological material via heat, which also calcified the gel into a monolithic and highly porous solid. ${ }^{15,17,21}$ This method of biotemplating silica materials has resulted in high degrees of structural similarity between the original template and the templated structure, including the ability to create functional actuators. ${ }^{17}$ However, the resulting material is brittle, the sample preparation phase relies on toxic chemicals, and only highly robust template materials (e.g., pine cones high in lignin ${ }^{17}$ ) can be used.

To create porous biotemplated materials from plants under mild synthesis conditions (i.e., without highly toxic chemicals or high heat ${ }^{21}$ ), while retaining the structure of the templated material across length scales, the research presented here used plants high in another common biopolymer, cellulose, in an environmentally friendly sol-gel biotemplating process. This biotemplating technique was inspired by methods used to create silica hydrogels that catalyze the gel transition by a change in $\mathrm{pH}$ and exposure to water. ${ }^{3,22,23}$ The templated material was Apium graveolens (Pascal celery), a vascular plant composed primarily of cellulose. Celery petioles have an elliptical cross-section containing vascular bundles, which are supported by structural parenchyma, a cellular structure. ${ }^{24}$ The vascular bundles are composed of xylem and transport fluid from the plant roots to leaves and phloem, and transport nutrients from the leaves to cells. ${ }^{13,24}$ This material was chosen because celery is common, inexpensive, and the cellular structure of the structural parenchyma and vascular structure of the xylem exist on the millimeter and micron scale, respectively. The biotemplating technique described in this article has four distinct and important features: (1) no highly toxic chemicals were used in the sample preparation phase, (2) geometrical similarity was retained on multiple length scales in functional structures, (3) the biotemplated material showed permeable porosity in gas flow testing, and withstood a sustained pressure drop, and (4) the mechanical strength of the final biotemplated material was increased compared to the original celery.

\section{MATERIALS AND METHODS}

As shown in Fig. 1a, biotemplating cellulosesilica composite materials was accomplished by sectioning samples of live celery, soaking the celery samples in acetic acid, placing the samples in a silica precursor, tetraethyl orthosilicate (TEOS; Acros Organics, Fair Lawn, NJ, USA), that introduced the inorganic phase and formed a network silica gel, which resulted in the final, cellulose-silica composite. Samples averaging $6.67 \mathrm{~mm}^{3}$ in volume were cut from stalks of celery procured from a local retailer and placed in $1 \% \mathrm{v} / \mathrm{v}$ glacial acetic acid (LabChem, Zelienople, PA, USA). The celery sections were allowed to soak for $72 \mathrm{~h}$ to break down the cellulose and acidify the sample (i.e., lower the $\mathrm{pH}$ from 6 to approximately 3). After the acetic acid soak, samples were removed from the acid and rinsed with tap water to remove excess acetic acid from the sample surface. The samples were then placed in TEOS (98\%) to soak for $48 \mathrm{~h}$, after which they were allowed to dry at room temperature for $72 \mathrm{~h}$. This procedure was inspired by previous work, creating keratin-silica hydrogels using acetic acid and TEOS for the fabrication of dressing materials for biomedical applications, ${ }^{22}$ and created samples referred to as cellulose-silica composites. This process was used to manufacture 16 cellulose-silica composites, 10 were used for mechanical testing, 4 for imaging, and 2 were used for single gas permeability testing.

Sections of approximately $5 \mathrm{~mm} \times 5 \mathrm{~mm} \times 10 \mathrm{~mm}$ from 4 cellulose-silica composites were removed for scanning electron microscopy (SEM) imaging in a vacuum environment (FEI Quanta 600 FG; Hillsboro, OR, USA). Control samples from the live celery stalk were taken from a location adjacent to those that underwent the biotemplating process to collect images of the pore structure. Live celery was used from an adjacent location to ensure that the imaged pore structure was as similar as possible in the cellulosesilica composites and the live celery samples so as to facilitate comparisons. Live samples were collected from celery stalks immediately before imaging, and were fully hydrated before being examined by SEM. The cellulose-silica composite materials were 


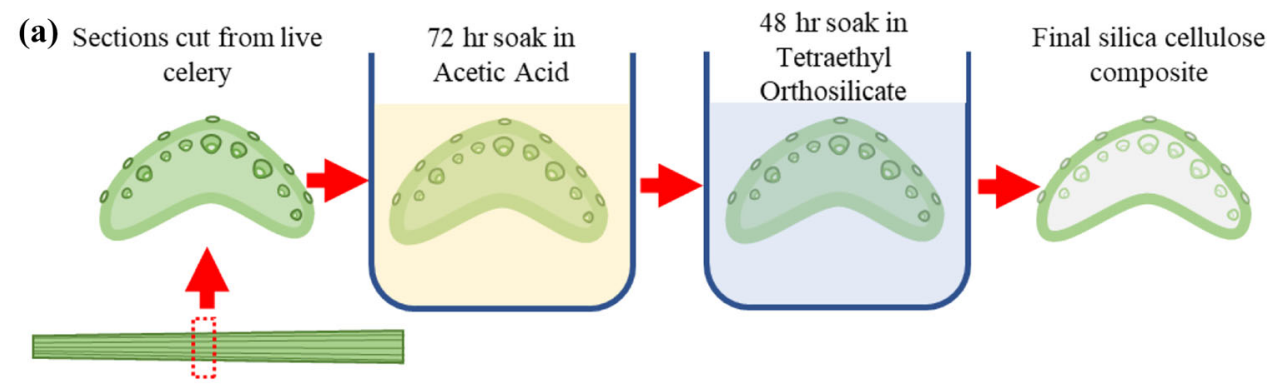

(b)

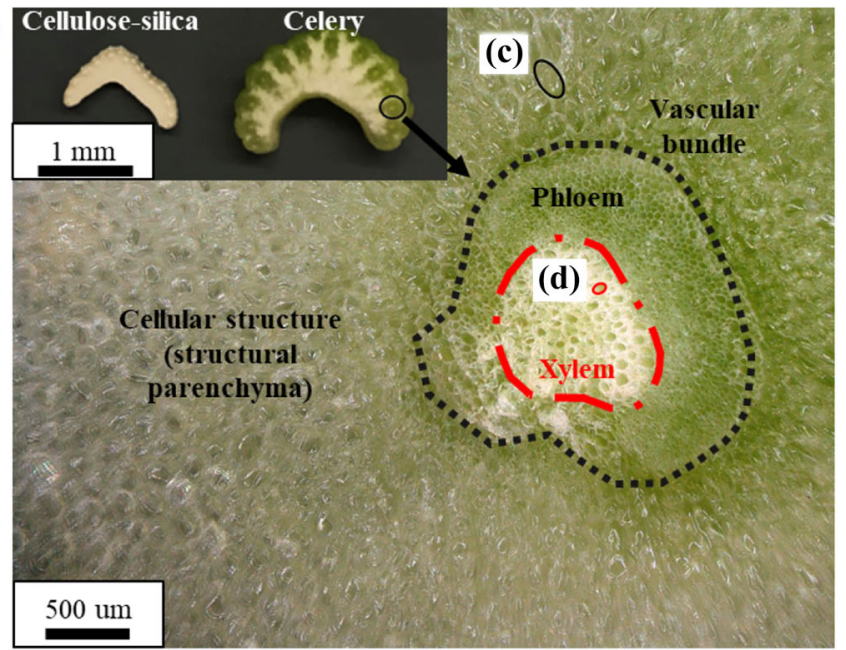

Fig. 1. (a) Process flow of the sol-gel biotemplating process. (b) Macroscale images of a cellulose-silica composite and a cross-section of live celery. The cellular structure of the structural parenchyma, the vascular bundle (black dashed line), the xylem (red dot-dashed line) and phloem are highlighted. The xylem transport fluid from celery plant roots to their leaves. Typical measurements for both the cellular structure and the xylem area are highlighted by (c) and (d), respectively.

imaged 8 times each. Four images of the structure of the cross-section on the millimeter scale were taken together with four images of the identifiable xylem on the micron scale. Measurements of the pore sizes were taken from both sets of imaged regions. These two scales were imaged due to their unique functions: the cell structure of the structural parenchyma provides structural strength to the celery stalks, and the xylem transports fluids through the stalk. Each of these structures are displayed in vivo in Fig. 1b, along with images of live celery cross-sections and cellulose-silica composites. Measurements of the area of the cells and xylem and their effective diameter (i.e., diameter calculated from their area assuming they were perfectly circular) and circularity (i.e., the ratio of the area and the perimeter squared multiplied by $4 \pi$ ) of all the collected images were carried out using ImageJ (National Institutes of Health, Bethesda, MD, USA). In total, 120 area and diameter measurements and 40 circularity measurements were collected from the cellular structure and xylem of the live celery and the templated cellulose-silica composites, respectively. The pore area measurements of the templated cellulose-silica composites were compared to the pore areas measured in the control live celery by a Tukey's Honest Significant Difference (HSD) test to quantify if statistically significant changes in pore area had occurred. For all statistical analyses, a confidence interval of $95 \%$ was used, i.e., a $p$ value less than 0.05 indicated a statistically significant difference existed between groups. These comparisons served as a quantitative metric of how well the biotemplating process retained structural similarity in the final cellulose-silica composites as compared to the original live celery.

In addition to imaging, 4 cellulose-silica composite sections and 4 live celery sections were subjected to energy-dispersive $\mathrm{x}$ ray spectroscopy (EDS). This allowed for the identification of the elements present in the imaged area to verify that a change in composition had occurred. EDS maps and spectra were collected in areas of the cellulose-silica composites and of the live celery samples composed of the xylem and the cellular structure. The spectra measured the composition of the imaged area as the atom $\%$ of elements present and the maps displayed the location of the elements identified by colored pixels. These measurements and maps provided quantitative proof of the composition change undergone in the biotemplating process and the distribution of the constituent elements. The EDS measurements were supplemented by x-ray diffraction (XRD) to identify any crystal structure present 
in the final biotemplated material. XRD measurements were performed using a Rigaku Miniflex 600 x-ray diffractometer (Rigaku, Tokyo, Japan).

The ultimate compressive strength (UCS) and elastic modulus $(E)$ of the cellulose-silica composites was investigated via compression testing. Cellulose-silica composite samples and live celery samples ( $n=10$ each) with an elliptical cross-section with an effective diameter of $15 \mathrm{~mm}$ and height of $4 \mathrm{~mm}$ were compressed to failure in an Instron 5967 load frame (Instron, Norwood, MA, USA) at a crosshead speed of $1 \mathrm{~mm} \mathrm{~min}{ }^{-1}$. The UCS and $E$ data collected from the cellulose-silica composites and the live celery samples were compared by a Tukey's HSD test to establish if a significant difference existed between the measurements. The stress-strain data and the material density was used to calculate the specific strength, specific stiffness, and energy absorption of the live celery and the cellulose-silica composites. This data was critical as an increase in mechanical properties of macroscale biotemplated materials has not been reported in previous biotemplating research. ${ }^{17,25}$

Single gas flow tests were performed using argon to measure the permeability and investigate the porosity of the cellulose-silica composites. A diagram of the gas flow fixture is shown in Fig. 2. Two samples of cellulose-silica composites were fixed on a membrane holder composed of a 3D-printed ABS plastic disc using a silicone adhesive (Loctite 59530, IDH 160809 Superflex ${ }^{\circledR}$ RTV Silicone Adhesive Sealant). The membrane holder was then placed in a filter holder (25 mm In-Line Filter Holder; Delrin Plastic, Pall), which was connected to the flow system. A digital mass flow controller (Alicat MCRQS 100SLPM-D Controller) was used to control the face velocity of the gas and a digital mass flow meter (Alicat MQS 100SLPM-D Mass Flowmeter) was used to observe the resulting flowrate and pressure from the sample. Gas flow tests were run with an empty filter holder and a filter holder with an empty membrane holder to isolate the pressure drop caused by the cellulose-silica composites from that of the filter holder and membrane holder, respectively. These data were used to calculate the permeability of the samples using Darcy's Law (Eq. 1) ${ }^{26}$

$$
Q=\frac{k A \Delta P}{\mu L}
$$

where $Q$ is the flow rate measured in $\mathrm{m}^{3} \mathrm{~s}^{-1}, k$ is the permeability measured in $\mathrm{m}^{2}, A$ is the crosssectional area of the sample exposed to gas measured in $\mathrm{m}^{2}, \Delta P$ is the effective pressure drop across the sample, $\mu$ is the dynamic (absolute) viscosity of the carrier gas (Ar), and $L$ is the thickness of the sample. The permeability data provided three insights into the cellulose-silica composites: (1) the nature of their porosity (as either open-cell or closed-cell), (2) the permeability of the porous structure, and (3) their mechanical resistance to pressure. These properties are important for potential filtration applications. ${ }^{27}$

\section{RESULTS AND DISCUSSION}

Figure 3 displays SEM images comparing the microstructure of the cellulose-silica composite and the live celery. In Fig. $3 \mathrm{~b}$, an inset provides a detailed view of the cellulose-silica composite microstructure in an area in which the structure is not obscured by the conductive coating to confirm the templating process filled microscale cavities. The cellulose-silica composite qualitatively shows a high degree of similarity to the structure of the celery in both the cellular structure and xylem on the macro- and microscale. The results of the collected area, effective diameter, and circularity measurements are presented in Table I. The measurements for both the cellulose-silica composites and the live celery were collected in either the cellular structure of the material cross-section or in the xylem. In both cases, the cellulose-silica composites and the live celery, the area and effective diameter measurements collected in the xylem were an order of magnitude smaller than those collected in the cellular structure. These measurements agree with intuition and previous literature, as the xylem in live celery are much smaller than the cellular structure due to their functional properties of fluid transport and strength, respectively. ${ }^{11,13}$ The area measurements collected from the cellular structure of the cellulose-silica composite and the live celery show no statistically significant differences in mean value (i.e., $p=0.0542$ ). This pairwise comparison does have a $p$ value very near statistical significance (i.e., $p<0.05$ ), where the live celery cellular structure displays a lower mean value (Table I). This is likely due to dehydration of the live celery samples caused by the vacuum environment of the SEM. The cellulose-silica composite

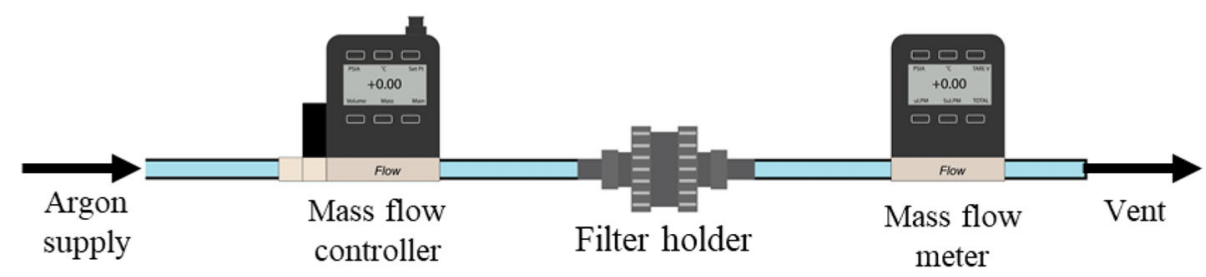

Fig. 2. The single gas flow testing apparatus composed of an argon supply, a digital mass flow controller used to control the face velocity of the gas, a filter holder, and a digital mass flow meter used to observe the resulting flowrate and pressure from the cellulose-silica composite. 
(a)

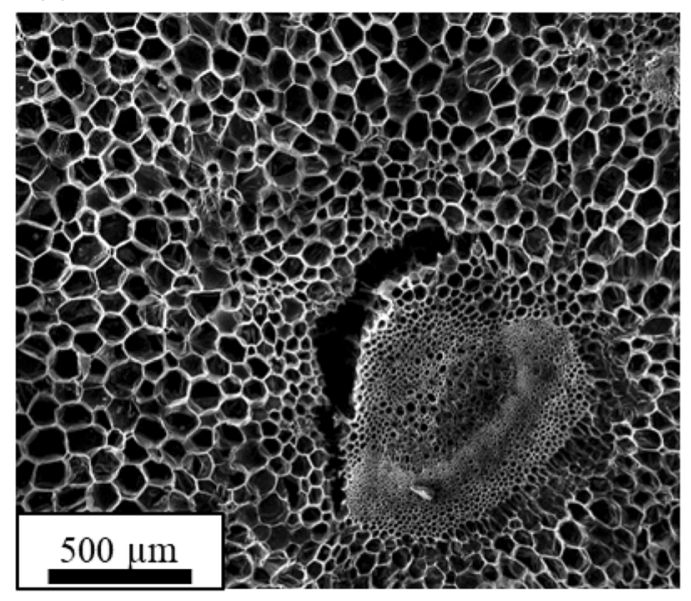

(b)

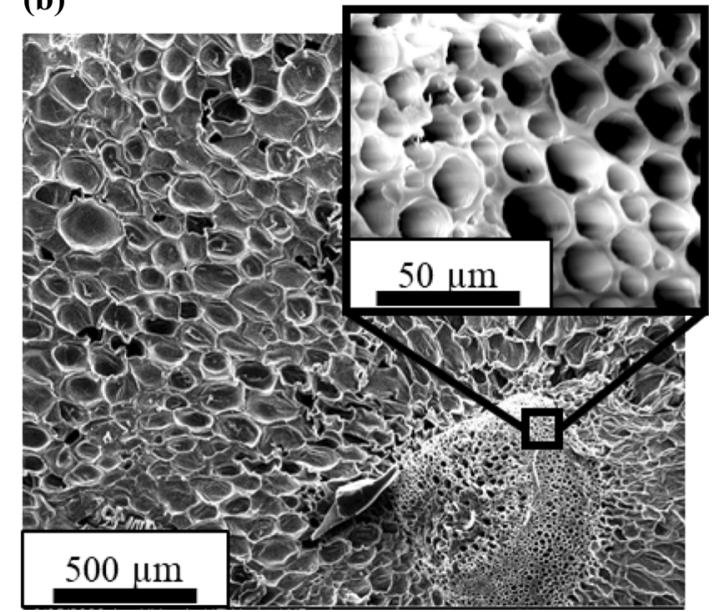

Fig. 3. SEM images of the structure of (a) live celery, (b) a biotemplated cellulose-silica composite and, inset, higher magnification of the biotemplated celery microstructure. The filled-in appearance of some of the cells in (b) is due to a conductive coating used to prevent charging during imaging.

Table I. Microstructural measurements of live celery and cellulose-silica composites

\begin{tabular}{|c|c|c|}
\hline & Cell area $\left(\mu \mathbf{m}^{2}\right)$ & Xylem area $\left(\mu \mathbf{m}^{2}\right)$ \\
\hline Live celery & $6612.88 \pm 3148.27^{\mathrm{a}}$ & $325.42 \pm 194.43^{b}$ \\
\hline \multirow[t]{2}{*}{ Cellulose-silica composite } & $7579.94 \pm 4983.19^{\mathrm{a}}$ & $442.63 \pm 227.47^{\mathrm{b}}$ \\
\hline & Cell diameter $(\mu \mathrm{m})$ & Xylem diameter $(\mu \mathrm{m})$ \\
\hline Live celery & $89.24 \pm 21.43^{\mathrm{a}}$ & $19.54 \pm 5.72^{\mathrm{b}}$ \\
\hline Cellulose-silica composite & Cell circularity & Xylem circularity \\
\hline Live celery & $0.9605 \pm 0.0389^{\mathrm{a}}$ & $0.9313 \pm 0.0742^{\mathrm{b}}$ \\
\hline Cellulose-silica composite & $0.9667 \pm 0.0630^{\mathrm{a}}$ & $0.9264 \pm 0.0638^{\mathrm{b}}$ \\
\hline
\end{tabular}

Measurements are provided as the mean \pm one standard deviation. Statistically significant differences are denoted by non-matching superscript letters.

materials underwent dehydration in the templating process, therefore the dehydration caused by the vacuum environment of the SEM ensured that the measurements were collected from materials in the same state of hydration, as hydration is known to effect the properties of natural materials. ${ }^{10}$ The area measurements of the xylem structure of the cellulose-silica composite and the live celery do not show statistically significant differences (i.e., $p=0.9899$ ). It is noteworthy that the measurements of the xylem and cellular structure do vary by a statistically significant amount for both the live celery and the cellulose-silica composites (i.e., $p>0.05$ ). The effective diameter measurements for both the cellular structure and xylem reflect the same lack of statistically significant difference between similar structures as was observed in the area measurements. The lack of statistically significant difference is quantitative evidence of the ability of this templating technique to template structures on multiple length scales, and preserve the structure of the templated material in the final cellulose-silica composite on these length scales. These properties have been identified in previous biotemplating work as critical for the retention of functional properties. ${ }^{6,14,17}$ The circularity measurements provide quantitative insight into the deformation of the geometry of the cellular structure and xylem in the templating process. When compared to the cellulose-silica composite cellular 
structure, the cellular structure of the live cells showed no statistically significant difference (i.e., $p=0.9696$ ), indicating that no appreciable deformation of the cellular structure geometry occurred. Similarly, when the circularity of the xylem in the cellulose-silica composites were compared to those of live celery, no statistically significant difference was observed (i.e., $p=0.9840$ ). Collectively, the results of the area, diameter, and circularity measurements prove a lack of deformation caused by the templating process and demonstrate that live celery was templated without the warping usually observed due to the residual stresses of the chemical processes associated with biotemplating. It is noteworthy that the difference in size observable in Fig. 1 is due to the dehydration of the cellulosesilica composites in the last step of the templating process. The lack of difference in the collected measurements is due to the dehydration of the live sample in the vacuum environment of the SEM. This highlights another advantage of this biotemplating process, specifically, the deformation which occurs is only due to dehydration and not to damage caused by the templating process, which does not rely on highly toxic chemicals. In previous biotemplating work that retained the functional properties of the template, macroscale shrinking of $30 \%$ was observed. ${ }^{17}$ It is important to note that this size change was caused by the templating process and not by dehydration, as the initial material was not hydrated. ${ }^{17}$

EDS data provided quantitative evidence of the change in material composition after the templating process. EDS maps along with the measured atom\% and the results of the XRD measurements are presented in Fig. 4. The live celery displayed a composition of 51 at.\% carbon and 45 at.\% oxygen, and only trace levels of silicon (i.e., $<1$ at.\%). This ratio of carbon and oxygen aligned with expectations, as the cell walls of vascular plants are primarily composed of cellulose, a biopolymer composed of carbon, hydrogen, and oxygen $\left(\mathrm{C}_{6} \mathrm{H}_{10} \mathrm{O}_{5}\right){ }^{11}$ The EDS spectra of the templated cellulose-silica composite materials revealed a composition of 21 at.\% carbon, 61 at.\% oxygen, and 16 at.\% silicon. The presence of silicon and oxygen was indicative of the presence of silica, ${ }^{28}$ and the carbon indicated that some cellulose had remained in the final material. Assuming all the measured silicon present is in the form of silica $\left(\mathrm{SiO}_{2}\right)$, approximately $59 \%$ of the oxygen present in the scans is bonded in the silica. Cellulose monomers $\left(\mathrm{C}_{6} \mathrm{H}_{10} \mathrm{O}_{5}\right)$ are much larger molecules, and are composed of a higher number of oxygen atoms. The relative percentage of oxygen atoms bonded in silica suggests a composition of approximately $49 \%$ silica and $51 \%$ cellulose in the cellulose-silica composite. The EDS maps presented in Fig. 4 display the distribution of the constituent elements found in both the live celery (Fig. 4a and b) and in the templated cellulose-silica composite (Fig. 4c and d). The EDS maps of the cellulose-silica composites also show silicon evenly distributed throughout the imaged area. Figure $4 \mathrm{e}$ displays the results of the XRD measurements, the lack of distinct peaks indicating that the silica phase is entirely amorphous. The XRD measurements are similar to those collected from previous biotemplated silica materials. ${ }^{6}$ As no crystal structure was detected by the XRD measurements, no other imaging techniques were employed to attempt to measure the crystal structure of the cellulosesilica composites. A crystal structure has been found in biotemplating processes that incorporate high heat. ${ }^{29}$ The combination of the EDS, XRD, and the measurements of the pore area, circularity, and effective diameter make it clear this new method of biotemplating successfully created cellulose-silica composite materials with an amorphous crystal structure and without significant deformation.

The measurements of both the $E$ and UCS collected from compression testing are presented in Fig. 5, along with representative stress-strain curves from three live celery and three cellulosesilica composite samples. The cellulose-silica composite materials exhibited statistically significant increases $\left(p<1 \times 10^{-8}\right)$ in both $E$ and UCS. The modulus of the cellulose-silica composites increased by $15.05 \times 10^{4} \%$ when compared to live celery. The UCS also increased by $3880 \%$ between the live celery and the cellulose-silica composites. The stress-strain data and the material density were used to calculate the specific strength and stiffness of the live celery and the cellulose-silica composites. The specific strength and stiffness are represented graphically in a Wegst-Ashby plot ${ }^{30}$ in Fig. 6, in which it is shown the cellulose-silica composites have a higher specific strength than previously biotemplated Ni-coated luffa sponges ${ }^{31}$ and most natural ceramics. ${ }^{30}$ The density of the cellulosesilica composites and the live celery were calculated to be $1.0534 \pm 0.4269 \mathrm{~g} / \mathrm{cm}^{3}$ and $1.0363 \pm 0.0609 \mathrm{~g} /$ $\mathrm{cm}^{3}$, respectively, and were not different by a statistically significant amount $(p=0.902)$. The energy absorption of the cellulose-silica composites and live celery were calculated to be $9.93 \pm 7.27 \mathrm{MPa}$ and $9.18 \pm 5.29 \mathrm{MPa}$, respectively, and did not vary by a statistically significant amount. Both the cellulose-silica composite and the live celery absorbed more energy than other highly porous biotemplated materials. ${ }^{31}$ The specific strength of cellulose-silica composites and live celery were calculated to be $3.573 \times 104 \pm 1.56 \times$ $102 \mathrm{MPa} /\left(\mathrm{g} / \mathrm{mm}^{3}\right)$ and $912.44 \pm 156.00 \mathrm{MPa} /(\mathrm{g} /$ $\left.\mathrm{mm}^{3}\right)$, respectively, an increase of $3815 \%$, which was statistically significant $(p=0.0018)$. The specific strength of cellulose-silica composites and live celery were calculated to be $2.391 \times 105 \pm 2.064 \times$ $105 \mathrm{MPa} /\left(\mathrm{g} / \mathrm{mm}^{3}\right)$ and $161.46 \pm 129.18 \mathrm{MPa} /(\mathrm{g} /$ $\mathrm{mm}^{3}$ ), respectively, which was statistically significant $\left(p=1 \times 10^{-8}\right)$. The increases in specific strength and stiffness are intuitive, based on the significant increases in $E$ and UCS observed in the 

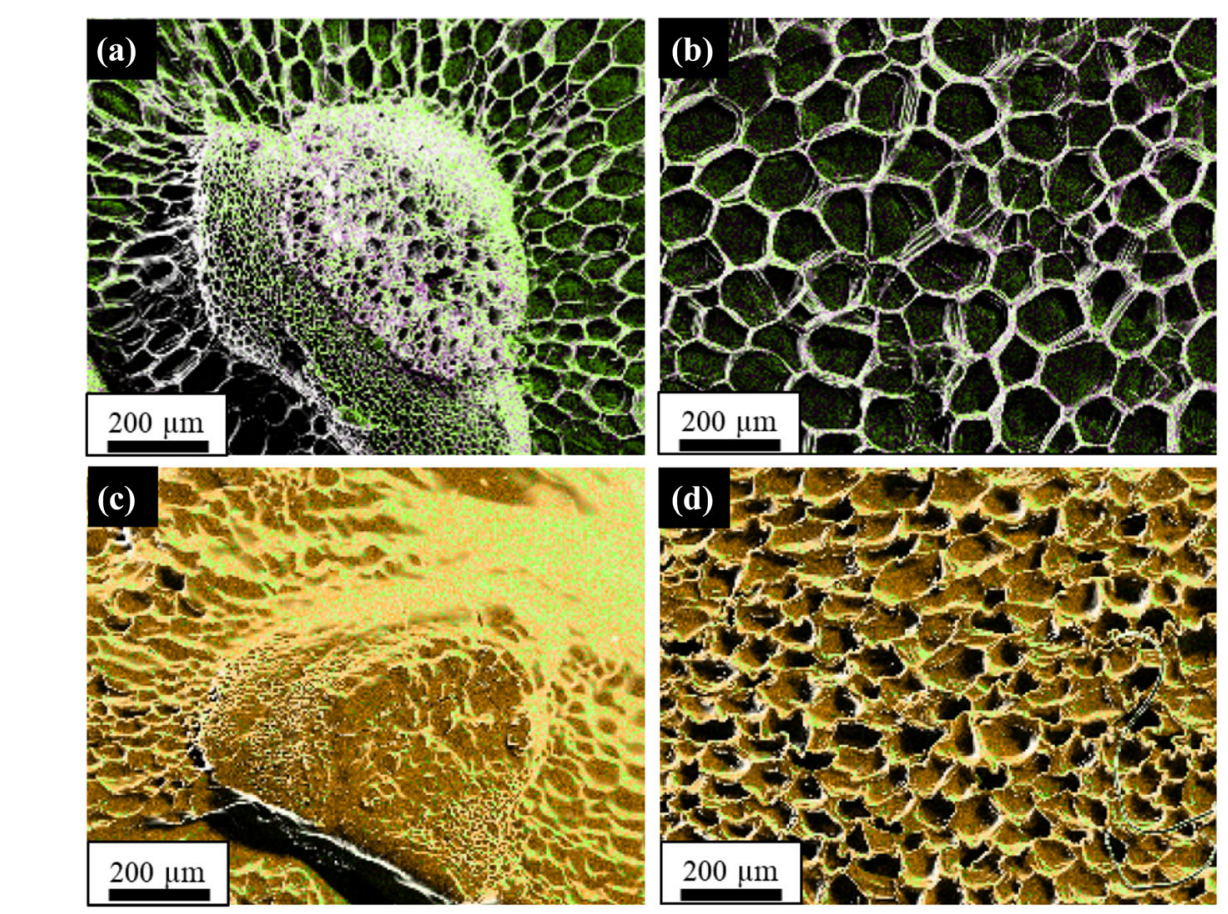

$\square$ Carbon $\square$ oxygen $\square$ Silicon

\begin{tabular}{|c|c|c|c|}
\hline & Carbon (atom \%) & Oxygen (atom \%) & Silicon (atom \%) \\
\hline Live celery & $51 \%$ & $45 \%$ & $<1 \%$ \\
\hline $\begin{array}{c}\text { Cellulose-silica } \\
\text { composite }\end{array}$ & $21 \%$ & $61 \%$ & $16 \%$ \\
\hline
\end{tabular}

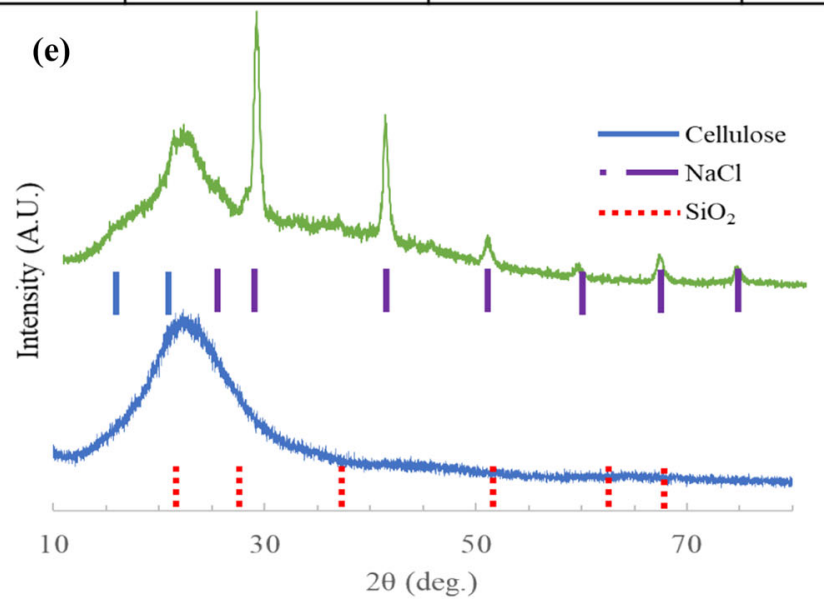

Fig. 4. Energy-dispersive x-ray spectroscopy (EDS) maps of (a) live celery xylem, (b) live celery cells, (c) cellulose-silica composite xylem, and (d) cellulose-silica composite cells displaying the constituent elements present and their distribution in the structure. The filled-in appearance of the cells in (c) and (d) is due to a conductive coating used to prevent charging during imaging. A table detailing the measured atom percentages of the material composition of the EDS maps is presented below the images. (e) Plot of the results of the XRD measurements; the top curve is the live celery and the bottom curve is the cellulose-silica composite.

cellulose-silica composites. These results provide quantitative evidence that this biotemplating technique results in an exceptionally stiff and strong material when compared to live celery and other biotemplated materials. ${ }^{25}$ This result is intuitive, as the cellulose-silica composite is similar to other mineralized biopolymers observed in natural materials, which are known to exhibit high mechanical strength. ${ }^{12,32}$

The Ar permeability of cellulose-silica composites was calculated from Eq. 1 at 6 different pressure differentials and was averaged from three sets of 


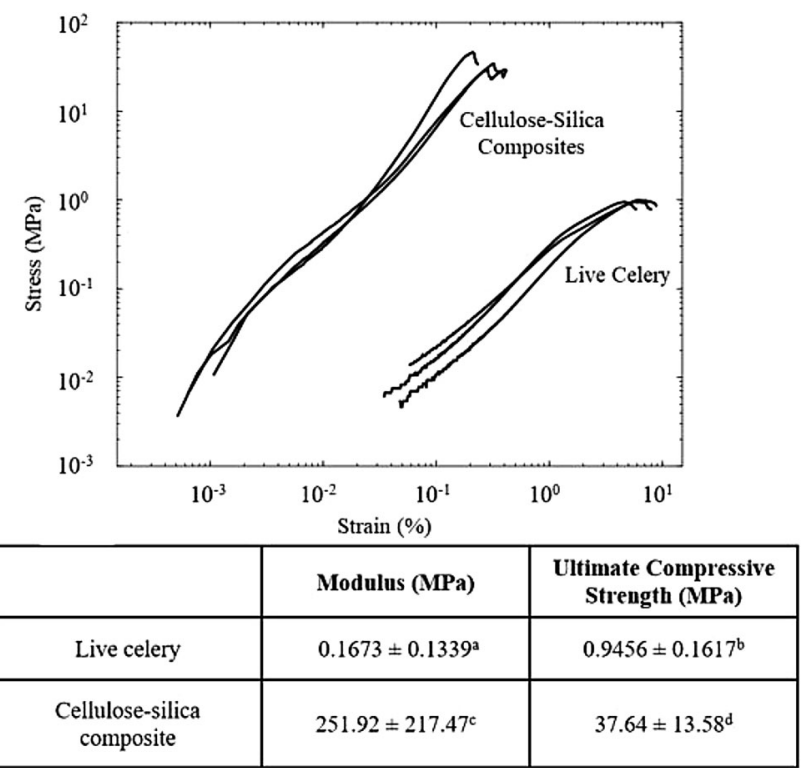

Fig. 5. Three representative stress-strain curves for both live celery and cellulose-silica composites, as well as a table of the modulus and ultimate compressive strength of live celery and cellulose-silica composites. It is noteworthy that, due to the differences in the stress-strain response, the data are presented on a logarithmic axis. Measurements are provided as the mean \pm one standard deviation $(n=10)$. Statistically significant differences are denoted by nonmatching superscript letters.

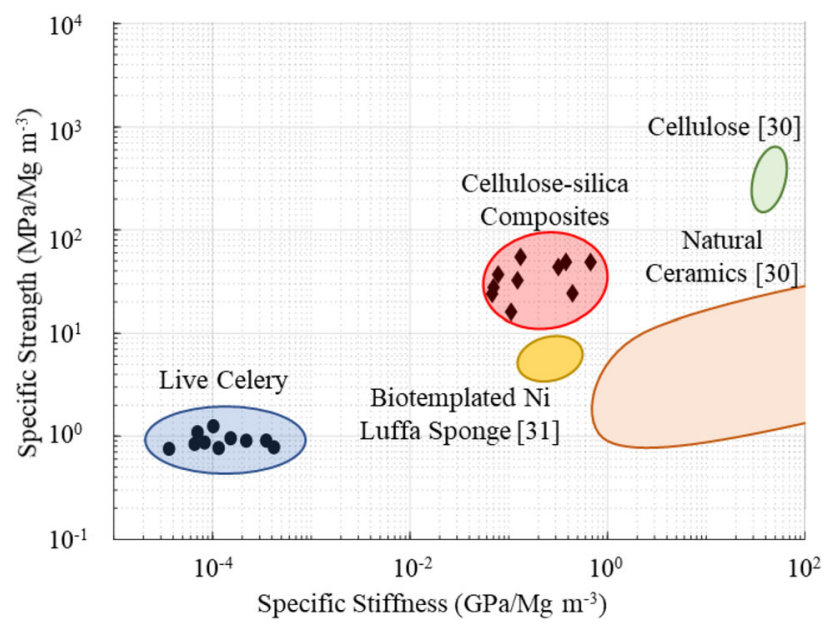

Fig. 6. A Wegst-Ashby plot of the specific strength $\left(\mathrm{MPa} / \mathrm{Mg} \mathrm{m}^{-3}\right)$ relative to the specific stiffness $\left(\mathrm{GPa} / \mathrm{Mg} \mathrm{m}^{-3}\right)$ of both live celery and cellulose-silica composites. For comparison, biotemplated $\mathrm{Ni}$ sponges, ${ }^{31}$ crystalline cellulose, ${ }^{30}$ and natural ceramics $^{30}$ are presented.

measurements that resulted in a mean value of $3.8 \times 10^{-13} \pm 0.3 \times 10^{-13} \mathrm{~m}^{2}$. A representative plot of the pressure drop as a function of flow through a membrane formed by a cellulose-silica composite is presented in Fig. 7. The linearity of the plot is indictive of sample durability (i.e., the sample did not deform significantly enough during testing to influence the flow characteristics). ${ }^{27,33}$ The non-zero offset indicates that there was no gas leakage in the apparatus. It is noteworthy that none of the control

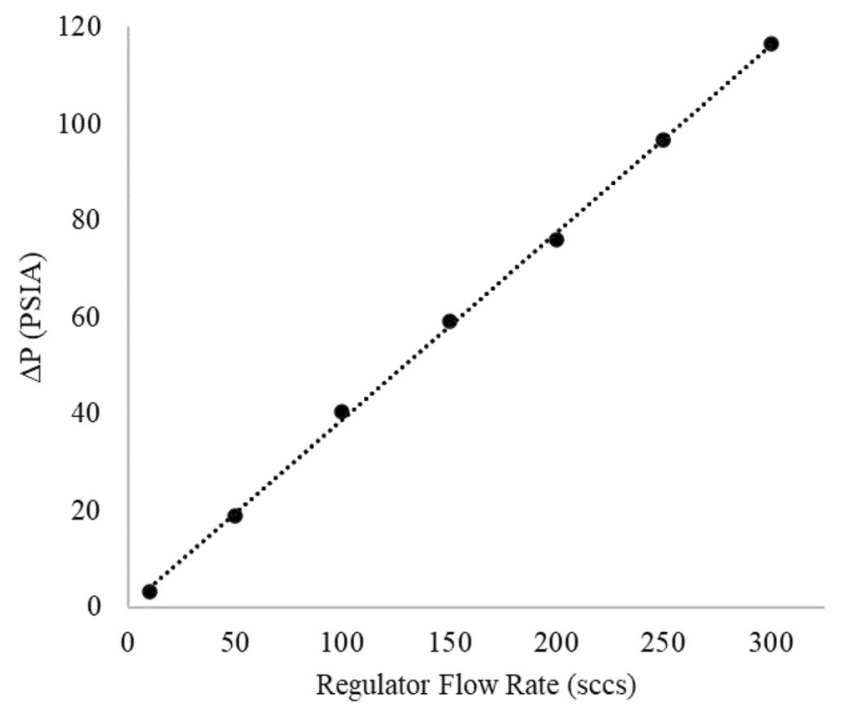

Fig. 7. Plot of the pressure drop as a function of flow through a membrane formed by a cellulose-silica composite. The linearity of the plot indicates the permeability of the membrane did not change with increasing differential pressure, as described in Ref. 25.

samples (i.e., desiccated native celery) were capable of maintaining a pressure differential without fracturing; however, the cellulose-silica composites were able to sustain the maximum allowable pressure for $15 \mathrm{~min}$ without fracture. The permeability is similar to that of other porous ceramics with low permeability (e.g., silica aerogel). ${ }^{26,34}$ The low permeability can be attributed to the structure of celery (Fig. 2). While the vascular bundle, composed of xylem and phloem, has a higher permeability due to its role in transporting fluids through the plant, the cross-sectional area of celery is dominated by parenchyma, which has a structure similar to that of a closed-cell foam (Table I). ${ }^{35}$ While the permeability is low, it is notable that any use of the complex structure of celery as a membrane for gas flow is only made feasible by the enhanced strength of the cellulose-silica composite provided through biotemplating.

This new method of biotemplating has three attractive features distinguishing it from other biotemplating techniques. By employing non-toxic chemicals in the first phase, this process eliminates many of the safety concerns of residual toxicity in the final samples, and it produces little toxic waste, making this process ecologically friendly. The use of inexpensive and safe materials (i.e., celery, acetic acid, and TEOS) also makes this process scalable. Second, the geometric measurements of the cellulose-silica composites provide quantitative evidence that this biotemplating process retains a high degree of geometric similarity between the live celery material and the cellulose-silica composite, and what deformation that does occur is caused by dehydration and not structural degradation, as has been observed in previous biotemplating reports. ${ }^{6,14,17}$ Finally, the mechanical properties of 
the cellulose-silica composite materials created in this research were orders of magnitude larger than those of the live celery and those reported in other biotemplating research. ${ }^{25}$ These cellulose-silica composites are also strong enough to maintain a pressure differential at different gas flow rates, opening up new applications for biotemplated materials as filtration membranes.

\section{CONCLUSION}

It has been shown that cellulose-silica composites biotemplated from live celery using a sol-gel technique retained the structure of the template material on multiple length scales, and exhibited both UCS and $E$ orders of magnitude higher than the template material without the use of highly toxic chemicals. These materials have potential applications as catalysts and for filtration. ${ }^{2,3}$ This work has the following conclusions:

- Catalyzing a sol-gel biotemplating process using a $\mathrm{pH}$ transition has resulted an ecologically friendly biotemplating technique for cellulosesilica composite materials with a pore and cell structure geometrically similar to live celery.

- It has been demonstrated that biotemplated cellulose silica composites possessed an elastic modulus and UCS $15.05 \times 10^{4} \%$ and $3880 \%$ higher than live celery, respectively.

- The resulting cellulose-silica composites demonstrated a permeability of $3.8 \times 10^{-13} \pm 0.3 \times$ $10^{-13} \mathrm{~m}^{2}$ under argon gas flow at elevated pressure.

\section{ACKNOWLEDGEMENTS}

The permeability tests were supported by the United States Department of Energy (DOE) under Contract DE-NE0008900.

\section{CONFLICT OF INTEREST}

The authors declare that they have no conflict of interests.

\section{REFERENCES}

1. Z.-G. Shi, Q.-Z. Guo, Y.-T. Liu, Y.-X. Xiao, and L. Xu, Mater. Chem. Phys. 126, 826 (2011).

2. F.-Q. Liu, L. Wang, Z.-G. Huang, C.-Q. Li, W. Li, R.-X. Li, W.-H. Li, and A.C.S. Appl, Mater. Interfaces 6, 4371 (2014).

3. R. Cademartiri, M.A. Brook, R. Pelton, and J.D. Brennan, J. Mater. Chem. 19, 1583 (2009).

4. W. Li, Q. Yue, Y. Deng, and D. Zhao, Adv. Mater. 25, 5129 (2013).

5. T. Amatani, K. Nakanishi, K. Hirao, and T. Kodaira, Chem. Mater. 17, 2114 (2005).
6. O. Paris, G. Fritz-Popovski, D. Van Opdenbosch, and C. Zollfrank, Adv. Funct. Mater. 23, 4408 (2013).

7. H. Nishihara, S.R. Mukai, D. Yamashita, and H. Tamon, Chem. Mater. 17, 683 (2005).

8. A.N. Khramov, and M.M. Collinson, Chem. Commun. 8, 767 (2001).

9. S.E. Naleway, M.M. Porter, J. McKittrick, and M.A. Meyers, Adv. Mater. 27, 5455 (2015).

10. P. Chen, A. Lin, Y. Lin, Y. Seki, A. Stokes, J. Peyras, E. Olevsky, M. Meyers, and J. Mckittrick, J. Mech. Behav. Biomed. Mater. 1, 208 (2008).

11. C. Beck, An Introduction to Plant Structure and Development, 2nd edn. (Cambridge: Cambridge University Press, 2010).

12. A.R. Studart, R. Libanori, and R.M. Erb, in Bio-Bioinspired Nanomaterials. ed. by D. Ruiz-Molina, F. Novio, and C. Roscini (Weinheim: Wiley-VCH, 2014), pp. 335-368.

13. J.A. Rosell, M.E. Olson, and T. Anfodillo, Curr. For. Rep. 3, 46 (2017).

14. O. Paris, I. Burgert, and P. Fratzl, MRS Bull. 35, 219 (2010).

15. C. Zollfrank, P. Cromme, M. Rauch, H. Scheel, M.H. Kostova, K. Gutbrod, S. Gruber, and D. Van Opdenbosch, Bioinspir. Biomim. Nanobiomater. 1, 13 (2012).

16. Q. Dong, H. Su, J. Xu, D. Zhang, and R. Wang, Mater. Lett. 61, 2714.(2007).

17. D. Van Opdenbosch, G. Fritz-Popovski, W. Wagermaier, O. Paris, and C. Zollfrank, Adv. Mater. 28, 5235 (2016).

18. S. Gravelle, and J. Dumais, J. Chem. Phys. 152, 014502 (2020).

19. I. Burgert, and P. Fratzl, Philos. Trans. R. Soc. Math. Phys. Eng. Sci. 367, 1541 (2009).

20. S. Deuerling, S. Kugler, M. Klotz, C. Zollfrank, and D. Van Opdenbosch, Adv. Mater. 30, 1703656 (2018).

21. T.-X. Fan, S.-K. Chow, and D. Zhang, Prog. Mater. Sci. 54, $542(2009)$

22. P. Kakkar, and B. Madhan, Mater. Sci. Eng. C 66, 178 (2016).

23. G. De, B. Karmakar, and D. Ganguli, J. Mater. Chem. 10, 2289 (2000).

24. F. Vilaine, J.-C. Palauqui, J. Amselem, C. Kusiak, R. Lemoine, and S. Dinant, Plant J. 36, 67 (2003).

25. D. Van Opdenbosch, G. Fritz-Popovski, J. Plank, C. Zollfrank, and O. Paris, Bioinspir. Biomim. 11, 065001 (2016).

26. J. Phalippou, T. Woignier, R. Sempéré, and P. Dieudonné, Mater. Sci. 20, 29 (2002).

27. B. Wang, A. Reifsnyder, I. Zharov, and K. Carlson, Microporous Mesoporous Mater. 278, 435 (2019).

28. P.B. Sarawade, J.-K. Kim, A. Hilonga, and H.T. Kim, J. Hazard. Mater. 173, 576 (2010).

29. J.-Y. Chen, C.-Y. Yang, and P.-Y. Chen, Mater. Sci. Eng. C 67, 85 (2016)

30. U.G.K. Wegst, and M.F. Ashby, Philos. Mag. 84, 2167 (2004).

31. S. Yin, H. Wang, J. Li, R.O. Ritchie, and J. Xu, J. Mech. Behav. Biomed. Mater. 94, 10 (2019).

32. J.W.C. Dunlop, and P. Fratzl, Annu. Rev. Mater. Res. 40, 1 (2010).

33. B. Hosticka, P.M. Norris, J.S. Brenizer, and C.E. Daitch, J. Non-Cryst. Solids 225, 293 (1998).

34. T. Ohji, Handbook of Advanced Ceramics (Amsterdam: Elsevier, 2013), pp 1131-1148.

35. L.J. Gibson, J. R. Soc. Interface 9, 2749 (2012).

Publisher's Note Springer Nature remains neutral with regard to jurisdictional claims in published maps and institutional affiliations. 